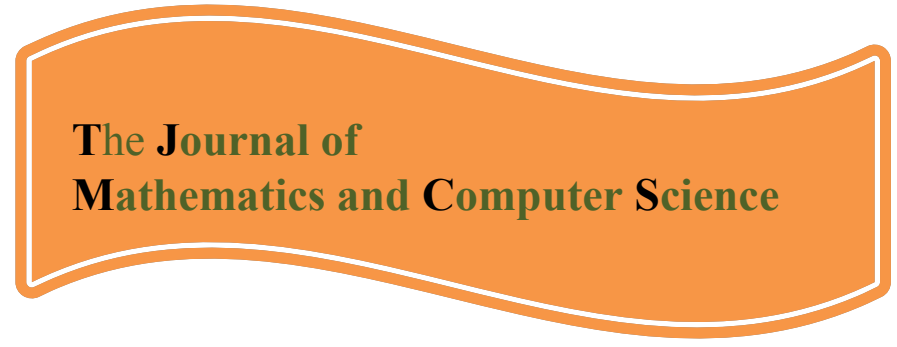

Available online at

http://www.TJMCS.com

The Journal of Mathematics and Computer Science Vol .5 No.2 (2012) 82-90

\title{
Union and intersection fuzzy subhypergroups
}

\author{
Esmail Ranjbar-Yanehsari \\ Department of Mathematics,Faculty of Sciences, Gorgan Branch,Islamic Azad University,Gorgan, Iran \\ es ranjbar@yahoo.com \\ Mohsen Asghari-Larimi \\ Department of Mathematics, GolestanUniversity, Gorgan, Iran \\ asghari2004@yahoo.com
}

Received: February 2012, Revised: November 2012

Online Publication: December 2012

\begin{abstract}
In this paper, some properties of union and intersection fuzzy subhypergroups are discussed. Generally, intersection family of fuzzy subhypergroups of a hypergroup is fuzzy subhypergroup, but, union family of fuzzy subhypergroups of a hypergroup is not fuzzy subhypergroup. We give some conditions such that this family is fuzzy subhypergroup.
\end{abstract}

Keywords: Hyperstructure; Fuzzy sets; Fuzzy subhypergroup.

2010 Mathematics Subject Classification: 20 N20.

\section{Introduction.}

Hyperstructures represent a natural extension of classical algebraic structures and they were introduced by the French mathematician F. Marty [10]. Algebraic hyperstructures are a suitable generalization of classical algebraic structures. In a classical algebraic structure, the composition of two elements is an element, while in an algebraic hyperstructure, the composition of two elements is a set. Since then, hundreds of papers and several books have been written on this topic, see [1, 2, $4,6,7,14]$. A recent book on hyperstructures [6] points out on their applications in fuzzy and rough set theory, cryptography, codes, automata, probability, geometry, lattices, binary relations, graphs and hypergraphs. Another book [7] is devoted especially to the study of hyperring theory. Several kinds of hyperrings are introduced and analyzed. The volume ends with an outline of applications in chemistry and physics, analyzing several special kinds of hyperstructures: e-hyperstructures and transposition hypergroups. The theory of suitable modified hyperstructures can serve as a mathematical background in the field of quantum communication systems. 
E. Ranjbar-Yanehsari, M. Asghari-Larimi / TJ MCS Vol .5 No. 2 (2012) 82-90

Given a set $\mathrm{H}$, a fuzzy subset of $\mathrm{H}$ (or a fuzzy set in $\mathrm{H}$ ) is, by definition, an arbitrary mapping $\mu: H \rightarrow[0,1]$ where $[0,1]$ is the usual interval of real numbers. This important concept of a fuzzy set has been introduced by Zadeh in [15]. Since then, many papers on fuzzy sets appeared showing the importance of the concept and its applications (cf., for example, $[3,5,6,11]$ ).

The study of the fuzzy algebraic structures has started in the pioneering paper of Rosenfeld [13] in 1971. Rosenfeld introduced the notion of fuzzy groups and showed that many results in groups can be extended in an elementary manner to develop the theory of fuzzy group. Since then the literature of various fuzzy algebraic concepts has been growing very rapidly. For example, the concept of a fuzzy ideal of a semigroup was introduced by Kuroki [8]. Liu in [9], and Mukherjee and Sen in [12], introduced and examined the notion of a fuzzy ideal of a ring.

This paper is structured as follows. After the introduction, in section 2, we recall some basic notions and results on hypergroups and fuzzy sets. In section 3, we study some properties of union fuzzy subhypergroups, give several examples and we estab-lish some characterization theorems. Finally, in Section 4, several characterization theorems are obtained, particularly about the relation between hyperstructure and union and intersection fuzzy subhypergroups.

\section{PRELIMINARIES}

Definition 2.1. ([4],[6]) Let $H$ be a non-empty set and $P^{*}(H)$ be the family of all non-empty subsets of $\mathrm{H}$. A hyperoperation or join operations a map $\odot: \mathrm{H} \times \mathrm{H} \rightarrow \mathrm{P}^{*}(\mathrm{H})$. If $(\mathrm{a}, \mathrm{b}) \in \mathrm{H} \times \mathrm{H}$, then its image under " $\odot$ " is denoted by $a \odot b$.

The join operation is extended to subsets of $\mathrm{H}$ in a natural way, so that $\mathrm{A} \odot \mathrm{B}$ is given by

$$
\mathrm{A} \odot \mathrm{B}=\mathrm{U}\{\mathrm{a} \odot \mathrm{b} \mid \mathrm{a} \in \mathrm{A}, \mathrm{b} \in \mathrm{B}\} .
$$

The notations $\mathrm{a} \odot \mathrm{A}$ and $\mathrm{A} \odot \mathrm{a}$ are used for $\{\mathrm{a}\} \odot \mathrm{A}$ and $\mathrm{A} \odot\{\mathrm{a}\}$ respectively.

Generally, the singleton $\{a\}$ is identified by its element $a$.

A non-empty set $\mathrm{H}$, endowed with a hyperoperation is called a hypergroupoid and it is denoted by $(\mathrm{H}, \odot)$. If

$$
x \odot(y \odot z)=(x \odot y) \odot z, \forall x, y, z \in \mathrm{H},
$$

then $(\mathrm{H}, \odot)$ is called a semihypergroup.

A hypergroupoid $(\mathrm{H}, \odot)$ is called a quasihypergroup, if $x \odot \mathrm{H}=\mathrm{H}=\mathrm{H} \odot x$, for all $x \in \mathrm{H}$.

Definition 2.2. A hypergroup is a semihypergroup and a quasihypergroup.

A fuzzy seton a non-empty set $\mathrm{H}$ is a function $\mu: \mathrm{H} \rightarrow[0 ; 1]$. We denote by $\mathrm{F}(\mathrm{H})$ the set all fuzzy subsets of $\mathrm{H}$. Let $\mu, \lambda \in \mathrm{F}(\mathrm{H})$, if $\mu(x) \leq \lambda(x)$ (resp. $\mu(x)<\lambda(x)$ ) for all $x \in \mathrm{H}$, then we say that $\mu$ is contained in $\lambda$ and we write $\mu \subseteq \lambda$ (resp. $\mu \subset \lambda$ ). Clearly, the inclusion relation $\subseteq$ is a partial ordering on $\mathrm{F}(\mathrm{H}$ ). Also, the mapping $1_{\mathrm{F}}: \mathrm{H} \rightarrow[0,1] \mid x \rightarrow 1_{\mathrm{F}}(x):=1$ is the greatest element of $\mathrm{F}(\mathrm{H})$, and the mapping $0_{\mathrm{F}}: \mathrm{H} \rightarrow[0,1] \mid x \rightarrow 0_{\mathrm{F}}(x):=0$ is the zero element of $\mathrm{F}(\mathrm{H})$ for all $x \in \mathrm{H}$, i.e., $0_{F} \subseteq \mu \subseteq 1_{F}$.

Define, $\mu \cup \lambda$ and $\mu \cap \lambda \in F(H)$ as follows:

$$
\begin{aligned}
& (\mu \cup \lambda)(x)=\max \{\mu(x), \lambda(x)\}, \\
& (\mu \cap \lambda)(x)=\min \{\mu(x), \lambda(x)\},
\end{aligned}
$$

for all $x \in \mathrm{H}$, then $\mu \cup \lambda$ and $\mu \cap \lambda$ are called the union and intersection of $\mu$ and $\lambda$, respectively. 
E. Ranjbar-Yanehsari, M. Asghari-Larimi / TJ MCS Vol .5 No. 2 (2012) 82-90

\section{RELATION BETWEEN HYPERGROUPS AND UNION FUZZY SUBGROUPS}

For any collection, $\left\{\mu_{\mathrm{i}}: \mathrm{i} \in \mathrm{I}\right\}$, of fuzzy subsets of $\mathrm{H}$, where $\mathrm{I}$ is a non-empty index set, the least upper $\cup_{i \in I} \mu_{i}$ and the greatest lower bound $\bigcap_{i \in I} \mu_{i}$ of the $\mu_{i}$ are given by $\left(\bigcup_{i \in I} \mu_{i}\right)(x)=\sup _{i \in I} \mu_{i}(x)$ and $\left(\cap_{\mathrm{i} \in \mathrm{I}} \mu_{\mathrm{i}}\right)(x)=\inf _{\mathrm{i} \in \mathrm{I}} \mu_{\mathrm{i}}(x)$ for all $x \in \mathrm{H}$, respectively. If $\mathrm{I}=\{1,2, \ldots, \mathrm{n}\}$, then we write

$$
\mathrm{U}_{\mathrm{i} \in \mathrm{I}} \mu_{\mathrm{i}}=\cup_{i \in \mathrm{I}}^{n} \mu_{\mathrm{i}}=\mu_{1} \cup \mu_{2} \cup \ldots \cup \mu_{\mathrm{n}}
$$

and

$$
\bigcap_{i \in \mathrm{I}}^{n} \mu_{\mathrm{i}}=\bigcap_{i=\mathrm{I}}^{n} \mu_{\mathrm{i}}=\mu_{1} \cap \mu_{2} \cap \ldots \mu_{\mathrm{n}} .
$$

Definition 3.1. ([6]) Let $(H, \odot)$ be a hypergroup and $\mu$ a fuzzy subset on $H$. We say that $\mu$ is a fuzzy subhypergroup on $\mathrm{H}$ if the following conditions hold:

1) $\forall(x, y) \in \mathrm{H}^{2}, \inf _{z \in x_{\odot} y} \mu(z) \geq \inf \{\mu(x), \mu(y)\}$

2) $\forall(x, a) \in H^{2}, \quad(y, z) \in H^{2}$ such that $x \in(a \odot y) \cap(z \odot a)$ and $\inf \{\mu(y), \mu(z)\} \geq \inf \{\mu(x), \mu(a)\}$.

Example 3.2. Let $H=\{0,1,2\}$, the hypergroupoid $(H, \odot)$ defined by the following table:

\begin{tabular}{c|ccc}
$\odot$ & 0 & 1 & 2 \\
\hline 0 & $\{0\}$ & $\{0,1\}$ & $\{0,2\}$ \\
1 & $\{0,1\}$ & $\{1\}$ & $\{1,2\}$ \\
2 & $\{0,2\}$ & $\{1,2\}$ & $\{2\}$
\end{tabular}

then $(\mathrm{H}, \odot)$ is a commutative hypergroup.

We defined fuzzy subset $\mu$ and $\lambda$ on $\mathrm{H}$ as follows:

$$
\mu(x)=\left\{\begin{array}{ll}
1, & x \in\{0,1\} \\
0, & x=2
\end{array} \quad, \quad \lambda(x)=\left\{\begin{array}{ll}
0, & x \in\{0,1\} \\
1, & x=2
\end{array} .\right.\right.
$$

It follows easily that $\mu$ and $\lambda$ are fuzzy subhypergroups of $H$.

Since $\mu(2)<\lambda(2)$ and $\lambda(0)<\mu(0)$, thus $\mu \nsubseteq \lambda$ and $\lambda \nsubseteq \mu$. Also, $(\mu \cup \lambda)(x)=1$ for all $x \in\{0,1,2\}$.

It is clear that, $\mu \cup \lambda$ is a fuzzy subhypergroup of $H$.

Remark 1. The fuzzy subsets $1_{F}$ and $O_{F}$ are fuzzy subhypergroup of $(H, \odot)$. Since $\forall(x, y) \in H^{2}$, $\inf _{z \in x}{ }_{\mathcal{O}^{y}} 1_{F}(z)=1 \geq \inf \left\{1_{F}(x), 1_{F}(y)\right\}$ and $\forall(x, a) \in H^{2}, \exists(y, z) \in H^{2}$ such that $x \in(a \odot y) \cap(z \odot a)$ [since $H$ is a hypergroup] and $\inf \left\{1_{F}(y), 1_{F}(z)\right\}=1 \geq 1=\inf \left\{1_{F}(x), 1_{F}(a)\right\}$. Similary we have for $0_{F}$.

Example 3.3. Let $\mathrm{H}=\{0,1,2,3,4,5\}$ be the cyclic group $\mathbb{Z}_{6}$ and $x \odot y=\mathrm{L}\{x, y\}$ where $\mathrm{L}\{x, y\}$ is the subgroup of $\mathrm{H}$ generated by $\{x, y\}$. Then the hypergroupoid $(\mathrm{H}, \odot)$ defined by the following table:

\begin{tabular}{c|cccccc}
$\odot$ & 0 & 1 & 2 & 3 & 4 & 5 \\
\hline 0 & $\{0\}$ & $\mathrm{H}$ & $\{0,2,4\}$ & $\{0,3\}$ & $\{0,2,4\}$ & $\mathrm{H}$ \\
1 & $\mathrm{H}$ & $\mathrm{H}$ & $\mathrm{H}$ & $\mathrm{H}$ & $\mathrm{H}$ & $\mathrm{H}$ \\
2 & $\{0,2,4\}$ & $\mathrm{H}$ & $\{0,2,4\}$ & $\mathrm{H}$ & $\{0,2,4\}$ & $\mathrm{H}$ \\
3 & $\{0,3\}$ & $\mathrm{H}$ & $\mathrm{H}$ & $\{0,3\}$ & $\mathrm{h}$ & $\mathrm{H}$
\end{tabular}


E. Ranjbar-Yanehsari, M. Asghari-Larimi / TJ MCS Vol .5 No. 2 (2012) 82-90

\begin{tabular}{c|cccccc}
4 & $\{0,2,4\}$ & $\mathrm{H}$ & $\{0,2,4\}$ & $\mathrm{H}$ & $\{0,2,4\}$ & $\mathrm{H}$ \\
5 & $\mathrm{H}$ & $\mathrm{H}$ & $\mathrm{H}$ & $\mathrm{H}$ & $\mathrm{H}$ & $\mathrm{H}$
\end{tabular}

then $(\mathrm{H}, \odot)$ is a commutative hypergroup.

If we define fuzzy subset $\mu$ and $\lambda$ on $\mathrm{H}$ as follows:

$$
\mu(x)=\left\{\begin{array}{ll}
1, & x \in\{0,2,4\} \\
0, & \text { o.w. }
\end{array}, \quad \lambda(x)= \begin{cases}\frac{3}{4}, & x \in\{0,3\} \\
1, & \text { o.w. }\end{cases}\right.
$$

Then $\mu$ and $\lambda$ are fuzzy subhypergroups of $H$.

Since $\mu(3)<\lambda(3)$ and $\lambda(2)<\mu(2)$, thus $\mu \nsubseteq \lambda$ and $\lambda \nsubseteq \mu$.

Since $(2,3) \in \mathrm{H}^{2}$ and

$$
\inf _{z \in 2} \odot^{3}(\mu \cup \lambda)(z)=\inf \{(\mu \cup \lambda)(0),(\mu \cup \lambda)(1),(\mu \cup \lambda)(2),(\mu \cup \lambda)(3),(\mu \cup \lambda)(4),(\mu \cup \lambda)(5)\}=\frac{1}{3}
$$

and

$$
\inf \{(\mu \cup \lambda)(2),(\mu \cup \lambda)(3)\}=\frac{3}{4}
$$

Thus

$$
\inf _{z \in 2 \odot_{\odot}}(\mu \cup \lambda)(z)<\inf \{(\mu \cup \lambda)(2),(\mu \cup \lambda)(3)\} .
$$

Therefore, following definition $3.1, \mu \cup \lambda$ is not a fuzzy subhypergroup of $\mathrm{H}$.

Corollary 3.4. In general, union of two fuzzy subhypergroup of a hypergroup is not a fuzzy subhypergroup.

Theorem 3.5. Let $\mu$ and $\lambda$ fuzzy subhypergroups of hypergroup $(H, \odot)$. Then $\mu \cup \lambda$ is a fuzzy subhypergroup of $H$ if $\mu \subseteq \lambda$ or $\lambda \subseteq \mu$.

Proof. Let $\mu \subseteq \lambda$ (similar argument is true for $\lambda \subseteq \mu$ ).

1) for all $(x, y) \in \mathrm{H}^{2}$, we have

$$
\begin{aligned}
\inf _{z \in x_{\odot} y}(\mu \cup \lambda)(z) & =\inf _{z \in x_{\odot}} y\{\mu(z) \vee \lambda(z)\} \\
& =\inf _{z \in x_{\odot} y} \lambda(z) \\
& \geq \inf \{\lambda(x), \lambda(y)\} \\
& =\inf \{\mu(x) \vee \lambda(x), \mu(y) \vee \lambda(y)\} \\
& =\inf \{(\mu \cup \lambda)(x),(\mu \cup \lambda)(y)\} .
\end{aligned}
$$

2) Since $\lambda$ is a fuzzy subhypergroup, thus $\forall(x, a) \in H^{2}, \exists(y, z) \in H^{2}$, such that $x \in(a \odot y) \cap(z \odot a)$ and $\inf \{\mu(y), \mu(z)\} \geq \inf \{\mu(x), \mu(a)\}$.

Consequently, $x \in(\mathrm{a} \odot \mathrm{y}) \cap(z \odot \mathrm{a})$ and

$$
\begin{aligned}
\inf \{(\mu \cup \lambda(y),(\mu \cup \lambda)(z)\} & =\inf \{\mu(y) \vee \lambda(y), \mu(z) \vee \lambda(z)\} \\
& =\inf \{\lambda(y), \lambda(z)\} \\
& \geq \inf \{\lambda(x), \lambda(a)\}
\end{aligned}
$$


E. Ranjbar-Yanehsari, M. Asghari-Larimi / TJ MCS Vol .5 No. 2 (2012) 82-90

$$
\begin{aligned}
& =\inf \{\mu(x) \vee \lambda(x), \mu(a) \vee \lambda(a)\} \\
& =\inf \{(\mu \cup \lambda)(x),(\mu \cup \lambda)(a)\} .
\end{aligned}
$$

Therefore $\mu \cup \lambda$ is a fuzzy subhypergroup.

Theorem 3.7. Let $I=\{1,2, \ldots, n\}$ and $\left\{\mu_{i}, i \in I\right\}$ be a family finite of fuzzy subhypergroups of hypergroup $H$. If $\mu_{1} \subseteq \mu_{2} \subseteq \ldots \subseteq \mu_{n}$, then, $\bigcup_{i=1}^{n} \mu_{i}$ is a fuzzy subhypergroup of $H$.

Proof. Let $\mu_{1} \subseteq \mu_{2} \subseteq \ldots \subseteq \mu_{\mathrm{n}}$. Then

1) For all $(x, y) \subseteq \mathrm{H}^{2}$, we have

$$
\begin{aligned}
\inf _{z \in x_{\odot} y}\left(\bigcup_{i=1}^{n}\right) \mu_{i}(z) & =\inf _{z \in x_{\odot} y}\left[\sup _{\mathrm{i} \in \mathrm{I}} \mu_{\mathrm{i}}(z)\right] \\
& =\inf _{z \in x_{\odot} y} \mu_{\mathrm{n}}(z) \\
& \geq \inf \left\{\mu_{\mathrm{n}}(x), \mu_{\mathrm{n}}(y)\right\} \\
& =\inf \left\{\sup _{\mathrm{i} \in \mathrm{I}} \mu_{\mathrm{i}}(x), \sup _{\mathrm{i} \in \mathrm{I}} \mu_{\mathrm{i}}(y)\right\} \\
& \left.\left.=\inf \left\{\bigcup_{i=1}^{n} \mu_{\mathrm{i}}\right)(x), \bigcup_{i=1}^{n} \mu_{\mathrm{i}}\right)(y)\right\} .
\end{aligned}
$$

2) For all $(x, a) \in H^{2}$, there exist $(y, z) \in \mathrm{H}^{2}$ such that $\mathrm{x} \in(\mathrm{a} \odot y) \cap(z \odot \mathrm{a})$ we have

$$
\begin{aligned}
\inf \left\{\sup _{\mathrm{i} \in \mathrm{I}} \mu_{\mathrm{i}}(y), \sup _{\mathrm{i} \in \mathrm{I}} \mu_{\mathrm{i}}(z)\right\} & =\inf \left\{\mu_{\mathrm{n}}(y), \mu_{\mathrm{n}}(z)\right\} \\
& \geq \inf \left\{\mu_{\mathrm{n}}(x), \mu_{\mathrm{n}}(\mathrm{a})\right\} \\
& =\inf \left\{\sup _{\mathrm{i} \in \mathrm{I}} \mu_{\mathrm{i}}(x), \sup _{\mathrm{i} \in \mathrm{I}} \mu_{\mathrm{i}}(\mathrm{a})\right\} \\
& =\inf \left\{\sup _{\mathrm{i} \in \mathrm{I}} \mu_{\mathrm{i}}(x), \sup _{\mathrm{i} \in \mathrm{I}} \mu_{\mathrm{i}}(\mathrm{a})\right\} \\
& \left.\left.=\inf \left\{\bigcup_{i=1}^{n} \mu_{\mathrm{i}}\right)(x), \bigcup_{i=1}^{n} \mu_{\mathrm{i}}\right)(\mathrm{a})\right\} .
\end{aligned}
$$

Hence by definition 3.1, $\mu_{\mathrm{i}}$ is a fuzzy subhypergroup.

Corollary 3.8. Let $I=\{1,2, \ldots\}$ and $\left\{\mu_{i}, i \in I\right\}$ be a family finite of fuzzy subhypergroups of hypergroup $H$. If there existn $\in \mathbb{N}$, where for all $i \geq n, \mu_{n+i}=\mu_{n}$, then, $\cup_{i=1}^{\infty} \mu_{\mathrm{i}}$ is a fuzzy subhypergroup of $H$.

Theorem 3.9. Let $I=\{1,2, \ldots\}$ and $\left\{\mu_{i}, i \in I\right\}$ be a family finite of fuzzy subhypergroups of hypergroup H. If $\mu_{1} \supseteq \mu_{2} \supseteq$..., then, $\bigcup_{i=1}^{\infty} \mu_{i}$ is a fuzzy subhypergroup of $H$.

Proof. The proof is similar to the proof of Theorem 3.7.

\section{RELATION BETWEEN HYPERGROUPS AND INTERSECTION FUZZY SUBGROUP}

Lemma 4.1. If $A$ be a non-empty of real number and there exist $\inf f_{x \in A} f(x)$ and $\inf f_{x \in A} g(x)$. Then,

$$
\left.\inf \left\{\inf _{x \in A} f(x), \inf _{x \in A} g(x)\right\}=\inf _{\inf }\{f(x), g(x)\}\right\} .
$$

Proof. Let $\inf _{x \in A} f(x)=\alpha, \inf _{x \in A} g(x)=\beta, \inf _{x \in A}\{f(x), g(x)\}=\gamma_{x}$ and $\alpha \leq \beta$.

Show that $\alpha=\inf \gamma_{\mathrm{x}}$. If $x \in A$ then $\alpha \leq \mathrm{f}(x)$ and $\beta \leq \mathrm{g}(x)$, hence $\alpha \leq \mathrm{f}(x), \alpha \leq \mathrm{g}(x)$ thus $\alpha \leq \inf \{\mathrm{f}(x), \mathrm{g}(x)\}=$ $\gamma_{x}$. Therefore, $\alpha \leq \inf _{x \in A} \gamma_{x}$.

Since $\alpha=\inf _{x \in A} \mathrm{f}(x)$ hence for all $\varepsilon>0$, there exist $x_{0} \in$ A such that: $\mathrm{f}\left(x_{0}\right) \leq \alpha+\varepsilon$. Since $\gamma_{x} \leq \mathrm{f}\left(x_{0}\right)$ therefore $\gamma_{x} \leq \mathrm{a}+\varepsilon$. Clearly $\inf _{x \in A} \gamma_{x} \leq \gamma_{x}$. Thus $\inf _{x \in A} \gamma_{x} \leq a+\varepsilon$. Consequently $\inf _{x \in A} \gamma_{x} \leq a$. Therefore, $\inf _{x \in A} \gamma_{x}=\alpha$.

Corollary 4.2. Let $\mu$ and $\lambda$ be fuzzy subhypergroup of $H$. Then for all $(x, y) \in H^{2}$ we have 
E. Ranjbar-Yanehsari, M. Asghari-Larimi / TJ MCS Vol .5 No. 2 (2012) 82-90

$$
\inf \left\{\inf _{z \in x_{\odot}} y(z), \inf _{z \in x_{\odot}} \lambda(z)\right\}=\inf \left\{\inf _{z \in x_{\odot}}\{\mu(z), \lambda(z)\}\right\}=\inf _{z \in x_{\odot} y}(\mu \cap \lambda)(z) .
$$

Example 4.3. Consider, again, the fuzzy subhypergroups $\mu$ and $\lambda$ defined in example 3.3. Then,

$$
(\mu \cap \lambda)(x)= \begin{cases}\frac{3}{4}, & x=0 \\ 0, & x \in\{1,3,5\} . \\ \frac{1}{3}, & x \in\{2,4\}\end{cases}
$$

Is follows easily that is a fuzzy subhypergroup of $\mathrm{H}$.

Theorem 4.4. If $\mu$ and $\lambda$ be fuzzy subhypergroup of $H$. Then, $\mu \cap \lambda$ is a fuzzy subhypergroup of $H$.

\section{Proof.}

1) Show that for all $(x, y) \in \mathrm{H}^{2}$, we have

$$
\inf _{z \in x_{\odot} y}(\mu \cap \lambda)(z) \geq \inf \{(\mu \cap \lambda)(x),(\mu \cap \lambda)(y)\} .
$$

The first solution: for all $(x, y) \in \mathrm{H}^{2}$, we have

$$
\inf _{z \in x_{\odot} y} \mu(z) \geq \inf \{\mu(x), \mu(y)\}
$$

and

$$
\inf _{z \in x_{\odot}} \lambda(z) \geq \inf \{\lambda(x), \lambda(y)\} .
$$

Suppose $\inf \{(\mu \cap \lambda)(x),(\mu \cap \lambda)(y)\}=\beta$. Then

$$
\beta \leq(\mu \cap \lambda)(x)=\mu(x) \wedge \lambda(x) \leq \mu(x)
$$

and

$$
\beta \leq(\mu \cap \lambda)(y)=\mu(y) \wedge \lambda(y) \leq \mu(y)
$$

hence, $\beta \leq \inf \{\mu(x), \mu(y)\}$.

Similarly, to be proved that $\beta \leq \inf \{\lambda(x), \lambda(y)\}$. Thus

$$
\inf _{z \in x_{\odot} y} \mu(z) \geq \inf \{(\mu \cap \lambda)(x),(\mu \cap \lambda)(y)\}
$$

and

$$
\inf _{z \in x_{\odot} Y} \lambda(z) \geq \inf \{(\mu \cap \lambda)(x),(\mu \cap \lambda)(y)\}
$$

Consequently,

$$
\inf \left\{\inf _{z \in x_{\odot}} y(z), \inf _{z \in x_{\odot}} \lambda(z)\right\} \geq \inf \{(\mu \cap \lambda)(x),(\mu \cap \lambda)(y)\} .
$$

Therefore, by corollary 4.2 , we have

$$
\inf _{z \in x_{\odot} y}(\mu \cap \lambda)(z) \geq \inf \{(\mu \cap \lambda)(x),(\mu \cap \lambda)(y)\} .
$$

2) The second solution: we claim that for all $(x, y) \in \mathrm{H}^{2}$ there is no member like $z_{0}$ in $x \odot y$ such that:

$$
(\mu \cap \lambda)\left(z_{0}\right)<\inf \{(\mu \cap \lambda)(x),(\mu \cap \lambda)(y)\} .
$$

Let there exist such a member.

Since $z_{0} \in x \odot y$ hence, $\mu\left(z_{0}\right) \geq \inf _{z \in x_{\odot} y} \mu(z) \geq \inf \{\mu(x), \mu(y)\}$. Therefore, $(\mu \cap \lambda)\left(z_{0}\right)=\mu\left(z_{0}\right) \wedge \lambda\left(z_{0}\right)$ 
E. Ranjbar-Yanehsari, M. Asghari-Larimi / TJ MCS Vol .5 No. 2 (2012) 82-90

$$
\begin{aligned}
& \geq \inf \{\inf \{\mu(x) \wedge \mu(y)\}, \inf \{\lambda(x) \wedge \lambda(y)\}\} \\
& =\inf \{\mu(x) \wedge \mu(y), \lambda(x) \wedge \lambda(y)\} \\
& =\inf \{\mu(x) \wedge \lambda(x), \mu(y) \wedge \lambda(y)\} \\
& =\inf \{(\mu \cap \lambda)(x),(\mu \cap \lambda)(y)\}
\end{aligned}
$$

Which it is in contradiction by hypothesis.

To prove the second condition defined 3.1, shown that the opposite is not true, i.e., the following is incorrect.

$\exists(x, \mathrm{a}) \in \mathrm{H}^{2}, \forall(y, \mathrm{z}) \in \mathrm{H}^{2}$ such that: $x \notin(\mathrm{a} \odot y) \cap(z \odot \mathrm{a})$

or

$$
\inf \{(\mu \cap \lambda)(y),(\mu \cap \lambda)(z)\}<\inf \{(\mu \cap \lambda)(x),(\mu \cap \lambda)(a)\} \cdot\left(^{*}\right)
$$

The first components $(*)$ is clearly not true (in contradiction with the definition of hypergroup). It is also clearly, where the second components $\left({ }^{*}\right)$ for some $y=x, z=a$ is not true.

Therefore, $\left(^{*}\right)$ is incorrect consequently, $\forall(x, \mathrm{a}) \in \mathrm{H}^{2}, \exists(y, z) \in \mathrm{H}^{2}$ such that: $x \in(\mathrm{a} \odot y) \cap(z \odot \mathrm{a})$ and $\inf \{(\mu \cap \lambda)(y),(\mu \cap \lambda)(z)\} \geq \inf \{(\mu \cap \lambda)(x),(\mu \cap \lambda)(a)\}$.

Thus, $\mu \cap \lambda$ is fuzzy subhypergroup of $H$.

Lemma 4.5. If $\left\{\mu_{i}: I \in I\right\}$ be a family of fuzzy sets of $H$ then, for all $(x, a) \in H^{2}$, we have

$$
\inf _{\mathrm{i} \in I}\left\{\inf \left\{\mu_{\mathrm{i}}(\mathrm{x}), \mu_{\mathrm{i}}(\mathrm{y})\right\}\right\}=\inf \left\{\inf _{\mathrm{i} \in \mathrm{I}} \mu_{\mathrm{i}}(x), \inf _{\mathrm{i} \in \mathrm{I}} \mu_{\mathrm{i}}(\mathrm{y})\right\} .
$$

Proof. Suppose $\inf \left\{\inf _{\mathrm{i} \in \mathrm{I}} \mu_{\mathrm{i}}(x), \inf _{\mathrm{i} \in \mathrm{I}} \mu_{\mathrm{i}}(y)\right\}=\alpha$ and $\inf _{\mathrm{i} \in \mathrm{I}}\left\{\inf _{\{}\left\{\mu_{\mathrm{i}}(x), \mu_{\mathrm{i}}(y)\right\}\right\}=\mathrm{b}$. Then, for all $\mathrm{i} \in \mathrm{I}, \mathrm{we}$ have $\beta \leq \inf \left\{\mu_{\mathrm{i}}(x), \mu_{\mathrm{i}}(y)\right\}$. Then $\beta \leq \mu_{\mathrm{i}}(x)$ and $\beta \leq \mu_{\mathrm{i}}(y)$. Thus $\beta \leq \inf _{\mathrm{i} \in \mathrm{I}} \mu_{\mathrm{i}}(x)$ and $\beta \leq \inf _{\mathrm{i} \in \mathrm{I}} \mu_{\mathrm{i}}(y)$. Then

$$
\beta \leq \inf _{\mathrm{i} \in I}\left\{\inf \left\{\mu_{\mathrm{i}}(x), \mu_{\mathrm{i}}(y)\right\}\right\}=\alpha .
$$

Also, $\alpha \leq \inf _{\mathrm{i} \in \mathrm{I}} \mu_{\mathrm{i}}(x)$ and $\alpha \leq \inf _{\mathrm{i} \in \mathrm{I}} \mu_{\mathrm{i}}(y)$. Thus for all $\mathrm{i} \in \mathrm{I}$, we have $\alpha \leq \mu_{\mathrm{i}}(x)$ and $\alpha \leq \mu_{\mathrm{i}}(y)$. Then $\alpha \leq \inf \left\{\mu_{\mathrm{i}}(x)\right.$, $\left.\mu_{\mathrm{i}}(y)\right\}$. Then

$$
\alpha \leq \inf \left\{\inf _{\mathrm{i} \in I}\left\{\mu_{\mathrm{i}}(x), \mu_{\mathrm{i}}(y)\right\}\right\}=\beta .
$$

Thus, $\alpha=\beta$.

Theorem4.6. if $\left\{\mu_{i}: i \in I\right\}$ be a family fuzzy subhypergroup of hypergroup $H$. Then $\cap_{i \in I} \mu_{i}$ is a fuzzy subhypergroup of $\mathrm{H}$.

\section{Proof.}

1) We claim that for all $(y, z) \in \mathrm{H}^{2}$, there is no member like $z_{0}$ in $x \odot y$ such that:

$$
\left(\cap_{i \in I} \mu_{i}\right)\left(z_{0}\right)<\inf \left\{\left(\cap_{i \in I} \mu_{i}\right)(x),\left(\cap_{i \in I} \mu_{i}\right)(y)\right\} .
$$

Let there exist such a member.

Since $z_{0} \in x \odot y$. Hence, for all $i \in I$ we have

$$
\mu_{\mathrm{i}}\left(z_{0}\right) \geq \inf _{z \in x_{\odot}} \mu_{\mathrm{i}}(z) \geq \inf \left\{\mu_{\mathrm{i}}(x), \mu_{\mathrm{i}}(y)\right\} .
$$

Consequently,

$$
\begin{aligned}
& \inf _{\mathrm{i} \in \mathrm{I}} \mu_{\mathrm{i}}\left(z_{0}\right) \geq \inf _{\mathrm{i} \in \mathrm{I}}\left\{\inf \left\{\mu_{\mathrm{i}}(x), \mu_{\mathrm{i}}(y)\right\}\right\} \\
& =\inf \left\{\inf _{\mathrm{i} \in \mathrm{I}} \mu_{\mathrm{i}}(x), \inf _{\mathrm{i} \in \mathrm{I}} \mu_{\mathrm{i}}(y)\right\} \\
& =\inf \left\{\left(\bigcap_{\mathrm{i} \in \mathrm{I}} \mu_{\mathrm{i}}\right)(x),\left(\ll_{\mathrm{i} \in \mathrm{I}} \mu_{\mathrm{i}}\right)(y)\right\} .
\end{aligned}
$$


E. Ranjbar-Yanehsari, M. Asghari-Larimi / TJ MCS Vol .5 No. 2 (2012) 82-90

Which it is in contradiction by hypothesis.

2) Show that, the following statement is in correct.

$\exists(x, \mathrm{a}) \in \mathrm{H}^{2}, \forall(y, z) \in \mathrm{H}^{2}$ such that: $x \notin(\mathrm{a} \odot y) \cap(z \odot \mathrm{a})$

Or

$\inf \left\{\left(\cap_{\mathrm{i} \in I} \mu_{\mathrm{i}}\right)(y),\left(\cap_{\mathrm{i} \in I} \mu_{\mathrm{i}}\right)(z)\right\}<\inf \left\{\left(\cap_{\mathrm{i} \in I} \mu_{\mathrm{i}}\right)(x),\left(\cap_{\mathrm{i} \in \mathrm{I}} \mu_{\mathrm{i}}\right)(\mathrm{a})\right\} .(\bullet)$

The first components $(\bullet)$ is clearly not true (in contradiction with the definition of hypergroup). It is also clearly, where the second components $(\bullet)$ for some $y=x, z=\mathrm{a}$ is not true.

Therefore, $(\bullet)$ is incorrect consequently,

$\forall(x, \mathrm{a}) \in \mathrm{H}^{2}, \exists(y, z) \in \mathrm{H}^{2}$ such that: $x \in(\mathrm{a} \odot y) \cap(z \odot \mathrm{a})$

and

$\inf \left\{\left(\cap_{\mathrm{i} \in \mathrm{I}} \mu_{\mathrm{i}}\right)(y),\left(\cap_{\mathrm{i} \in \mathrm{I}} \mu_{\mathrm{i}}\right)(z)\right\} \geq \inf \left\{\left(\cap_{\mathrm{i} \in \mathrm{I}} \mu_{\mathrm{i}}\right)(x),\left(\cap_{\mathrm{i} \in \mathrm{I}} \mu_{\mathrm{i}}\right)(\mathrm{a})\right\}$.

Thus, $\bigcap_{\mathrm{i} \in \mathrm{I}} \mu_{\mathrm{i}}$ is fuzzy subhypergroup of $\mathrm{H}$.

Definition 4.7. Let $\mu$ be fuzzy subhypergroup of $\mathrm{H}$. Then fuzzy subhypergroup generated by $\mu$ denoted by $\langle\mu\rangle$, is the smallest fuzzy subhypergroup of $H$ which contains $\mu$. That is

$<\mu>=\cap\{\lambda \in \mathrm{F}(\mathrm{H}): \lambda$ is fuzzy subhypergroup and $\mu \subseteq \lambda\}$.

By Theorem 4.6, we have the following corollary.

Corollary 4.8. Let $\mu \in F(H)$. Then $<\mu>$ is a fuzzy subhypergroup of $H$.

Acknowledgement. The author is highly grateful to the referees for their constructive suggestions for improving the paper.

\section{References}

[1] M. Asghari-Larimi, B. Davvaz, Hyperstructures associated to arithmetic functions, ArsCombitoria, 97 (2010) 51-63.

[2] M. Asghari-Larimi, V. Leoreanu-Fotea, A connection between hypergroupoids and L-Fuzzy Sets of Type 2, Italian J. of Pure and Appl. Math., 26 (2009), 207-216.

[3] P. Corsini, A new connection between hypergroups and fuzzy sets. Southeast, Asian Bull.Math. 27 (2003) 221-229.

[4] P. Corsini, Prolegomena of hypergroup theory, Second edition, Aviani editor, 1993.

[5] P. Corsini, Join spaces, power sets, fuzzy sets, Algebraic hyperstructures and applications (Iasi, 1993), 45-52, Hadronic Press, Palm Harbor, FL, 1994.

[6] P. Corsini, V. Leoreanu-Fotea, Applications of hyperstructure theory, Advances in Mathematics, Kluwer Academic Publishers, Dordrecht, 2003.

[7] B. Davvaz, V. Leoreanu-Fotea, Hyperring theory and Applications, Hadronic Press, Inc,115, Palm Harber, USA, (2009).

[8] N. Kuroki, On fuzzy ideals and fuzzy bi-ideals in semigroups, Fuzzy Sets and Systems 5 (1981), 203-215. 
E. Ranjbar-Yanehsari, M. Asghari-Larimi / TJ MCS Vol .5 No. 2 (2012) 82-90

[9] W.-J. Liu, Fuzzy invariant subgroups and fuzzy ideals, Fuzzy Sets and Systems 8 (1982),133139.

[10] F. Marty, Sur une generalization de la notion de groupe, 8th Congress Math. Scandenaves,Stockholm, (1934) 45-49.

[11] J.N. Mordeson, K.R. Bhutani, A. Rosenfeld,Fuzzy Group Theory, World Scientific, Singapore, 2005.

[12] T.K. Mukherjee, M.K. Sen, On fuzzy ideals of a ring (1), Fuzzy Sets and Systems 21 (1987),99-104.

[13] A. Rosenfield, Fuzzy groups, J. Math. Appl. 35 (1971), 512-517.

[14] T. Vougiouklis, Hyperstructures and their representations, Hadronic Press, Inc, 115, PalmHarber, USA, (1994).

[15] L.A. Zadeh, Fuzzy Sets, Inform and Control 8 (1965) 338-353. 